\title{
Improving Smoothing Efficiency of Rigid Conformal Polishing Tool Using Time-Dependent Smoothing Evaluation Model
}

\author{
Chi SONG ${ }^{1,3^{*}}$, Xuejun ZHANG ${ }^{1,2}$, Xin ZHANG ${ }^{1,2}$, Haifei $\mathrm{HU}^{1,2}$, and Xuefeng ZENG ${ }^{1,2}$ \\ ${ }^{1}$ Changchun Institute of Optics, Fine Mechanics and Physics, Chinese Academy of Sciences, Changchun, 130033, China \\ ${ }^{2}$ Key Laboratory of Optical System Advanced Manufacturing Technology, Chinese Academy of Sciences, Changchun, \\ 130033, China \\ ${ }^{3}$ University of Chinese Academy of Sciences, Beijing, 100049, China \\ *Corresponding author: Chi SONGＥ-mail: songchi7787@126.com
}

\begin{abstract}
A rigid conformal (RC) lap can smooth mid-spatial-frequency (MSF) errors, which are naturally smaller than the tool size, while still removing large-scale errors in a short time. However, the RC-lap smoothing efficiency performance is poorer than expected, and existing smoothing models cannot explicitly specify the methods to improve this efficiency. We presented an explicit time-dependent smoothing evaluation model that contained specific smoothing parameters directly derived from the parametric smoothing model and the Preston equation. Based on the time-dependent model, we proposed a strategy to improve the RC-lap smoothing efficiency, which incorporated the theoretical model, tool optimization, and efficiency limit determination. Two sets of smoothing experiments were performed to demonstrate the smoothing efficiency achieved using the time-dependent smoothing model. A high, theory-like tool influence function and a limiting tool speed of 300 RPM were obtained.
\end{abstract}

Keywords: Optics design and fabrication; optics fabrication; polishing

Citation: Chi SONG, Xuejun ZHANG, Xin ZHANG, Haifei HU, and Xuefeng ZENG, "Improving Smoothing Efficiency of Rigid Conformal Polishing Tool Using Time-Dependent Smoothing Evaluation Model," Photonic Sensors, 2017, 7(2): $171-181$.

\section{Introduction}

Large aspheric optical surfaces can be precisely manufactured using computer-controlled optical surfacing (CCOS). For next-generation largeaperture and high-resolution imaging optical systems such as the thirty meter telescope (TMT) and giant magellan telescope (GMT) [1, 2], correcting mid-spatial-frequency (MSF) errors on the optical surfaces is very important. Failure to control the MSF characteristics yields reduced optical performance due to the resultant MSF errors, which are directly related to the point-spreadfunction sharpness [3].

Two different approaches to controlling MSF errors exist: directed figuring and natural smoothing. The directed figuring of small-scale errors requires small tools, which in turn requires a long polishing run time, high-accuracy optical metrology, and high-accuracy tool positioning. A large polishing tool can naturally correct MSF errors smaller than the tool size, while also removing large-scale errors within a short time period. However, smoothing MSF errors from aspheric mirrors using large tools

Received: 3 November 2016 / Revised: 10 January 2017

(C) The Author(s) 2017. This article is published with open access at Springerlink.com

DOI: $10.1007 / \mathrm{s} 13320-017-0400-\mathrm{x}$

Article type: Regular 
is challenging, because the curvature changes on the surface require tools with sufficient compliance to fit the surface, but with sufficient rigidity to realize natural smoothing.

In 2010, Kim and Burge developed a rigid conformal (RC) lap using a visco-elastic non-Newtonian fluid to overcome the conflict between the desired rigidity and flexibility for large polishing laps [4]. Compared with active stress laps [5] and semi-flexible tools [6], an RC lap provides numerous advantages, such as ease of large-scale manufacture, superior surface roughness, and a highly stable theory-like tool influence function (TIF). However, the RC-lap smoothing efficiency performance is poorer than expected [7].

To quantify the smoothing effect, various mathematical models have been developed. For example, Brown et al. proposed a smoothing model for an elastic-backed flexible lapping belt in 1981 [8]. Subsequently, Jones simulated MSF error evolution based on a simple linear parametric model [9], and Mehta and Reid later proposed the classic Bridging model to study the smoothing effect, which is based on the theory of elasticity [10]. Tuell et al. then improved the Bridging model using the spatial Fourier decomposition method [11]. Later, Kim et al. introduced a parametric smoothing model based on the simplified Bridging model to describe smoothing effects [12]; this parametric smoothing model was further verified by Shu et al. using a correlation-based model [13]. Shu et al. also noted that the model presented by Kim et al. neglected the instantaneous property and therefore constructed a new model based on the Bridging model [14]. This new model discloses the exponential decay of the MSF errors with time during smoothing. Finally, Nie et al. constructed a generalized numerical pressure distribution model to solve the superposition of innumerable sinusoidal errors with different frequencies and amplitudes through finite element analysis (FEA) [15].

As indicated above, the majority of the past works have focused on the construction of models to quantitatively describe the smoothing effect. However, no explicit guidance has been developed to specify methods through which the smoothing efficiency can be improved. For instance, the parametric smoothing model reveals that the smoothing efficiency is related to the tool stiffness. However, if the smoothing time is considered, a greater number of factors can influence the smoothing efficiency.

In this paper, we present a time-dependent smoothing model containing specific factors directly related to the smoothing efficiency, which is derived from the parametric smoothing model and Preston equation. Based on this model, we can maximize the RC-lap smoothing performance. The remainder of the paper is organized as follows. In Section 2, we briefly introduce the general information on the RC lap and the parametric smoothing model. In Section 3 , we propose a strategy to improve the smoothing efficiency, which incorporates the theoretical model, tool optimization, and efficiency limit determination. In Section 4, we apply the above principles to actual smoothing experiments, and present the results. The conclusions are given in Section 5.

\section{RC lap and parametric smoothing model}

(1) RC lap and smoothing effect

The RC lap uses a non-Newtonian fluid (SillyPutty $^{\mathrm{TM}}$ ) as a medium, which conforms to an aspheric surface while generating a naturally smoothing effect. Having rigidity and viscosity intermediate between a solid and a liquid, the RC lap has the advantages of both rigid and compliant tools. When the tool is slowly travelling along the tool path, it acts like a compliant tool over a large time scale. However, in the short term, the RC lap behaves like a rigid tool, as a result of the tool motion and bumpy surface.

The basic principles of the RC-lap smoothing effect are straightforward. As the RC lap has a flow 
characteristic, it can fully deform in response to MSF errors. The MSF-error peaks have higher pressure than the valleys, because of the elastic property of the non-Newtonian fluid. The MSF errors then converge, because of the nonuniform material removal. The polishing pressure distribution between the RC lap and workpiece induced by a particular spatial frequency feature for the one-dimensional (1D) case is shown in Fig. 1. Note that the specific tool structure is presented in Section 3(3).

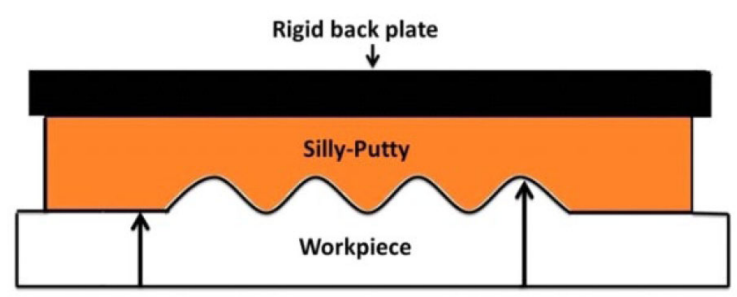

Nominal polishing pressure High local polishing pressure on peak

Fig. 1 RC-lap polishing pressure distribution induced by sinusoidal error.

(2) Parametric smoothing model

The parametric smoothing model can be used to describe the smoothing effect for visco-elastic polishing tools [12]. The dimensionless smoothing factor $S F$ is defined as the ratio of $\Delta \varepsilon$ to $\Delta Z$, where $\Delta \varepsilon$ is the difference between the peak-to-valley (PV) magnitude of a particular spatial frequency error before and after smoothing, and $\Delta Z$ is the corresponding change in the nominal removal depth. The linear relationship between $S F$ and the initial surface error $\varepsilon_{\text {ini }}$ can be expressed as

$$
S F=\frac{\Delta \varepsilon}{\Delta Z}=k \cdot\left(\varepsilon_{\text {ini }}-\varepsilon_{0}\right)
$$

where $\varepsilon_{0}$ is a fixed value indicating the final smoothing limit, and $k$ is the sensitivity to $\varepsilon_{\text {ini. }}$

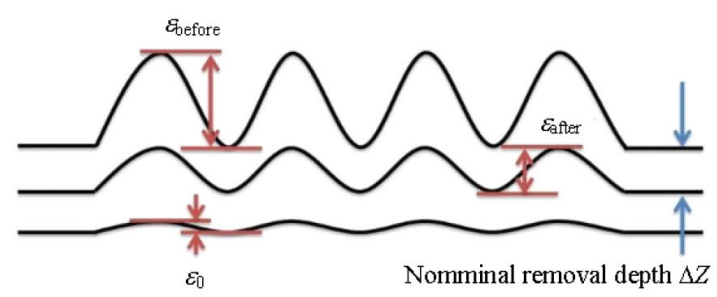

Fig. 2 Before $\varepsilon_{\text {before}}$, after $\varepsilon_{\text {after }}$, and final $\varepsilon_{0}$ smoothing profiles for sinusoidal error.
As shown in Fig. $2, \varepsilon_{0}, \Delta \varepsilon$, and $\Delta Z$ can be measured experimentally during smoothing, and the smoothing efficiency $k$ can be obtained by fitting the measurement results. The $S F$ function slope is proven to be related to the tool stiffness $\kappa_{\text {total }}$. However, the $k$ defined in this model neglects the instantaneous property. It has been shown that the MSF errors decay exponentially with time during smoothing [14]. Unfortunately, the smoothing rate is not yet well defined.

\section{Smoothing-efficiency $k$ improvement strategy}

(1) Theoretical model

In order to derive an explicit equation for $k$ improvement, we now present a time-dependent smoothing model, which contains specific factors related to the smoothing rate.

For the orbital motion, the instantaneous lap velocity $V(\mathrm{~mm} / \mathrm{s})$ can be obtained from the stroke speed $\Omega(\mathrm{RPM})$ and the orbital radius $A(\mathrm{~mm})$, such that

$$
V=\frac{2 \pi \cdot \Omega \cdot A}{60} .
$$

Based on the dynamic mechanical properties of Silly-Putty, the storage modulus $E^{\prime}$ is a function of the applied stress frequency $f(\mathrm{~Hz})$, as shown in Fig. 3 [16]. Because $f$ is determined by the local features under the tool motion, it can be expressed as

$$
f=V \cdot \xi=2 \pi \cdot \Omega \cdot A \cdot \xi / 60
$$

where $\xi\left(\mathrm{mm}^{-1}\right)$ is the spatial frequency of a sinusoidal error.

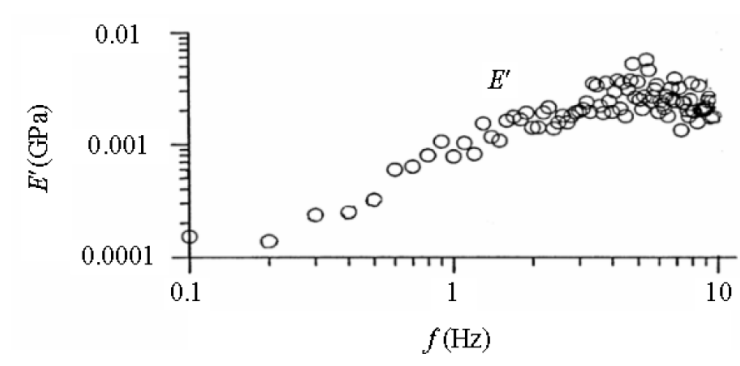

Fig. 3 Silly-putty storage modulus $E^{\prime}$ as a function of applied stress frequency $f$. 
According to the parametric smoothing model derivation process, the compressive stiffness of the entire tool $\kappa_{\text {total }}$ and the slope of the $S F$ function, which corresponds to $k$, can be expressed as

$$
k=\frac{\frac{1}{\kappa_{\text {total }}}=\frac{1}{\kappa_{\text {elastic }}(f)}+\frac{1}{\kappa_{\text {others }}}}{P_{\text {nominal }} \cdot\left[\frac{1}{\kappa_{\text {elastic }}(f)}+\frac{1}{\kappa_{\text {others }}}\right]}
$$

where $\kappa_{\text {elastic }}$ is the elastic stiffness of the Silly-Putty, $\kappa_{\text {others }}$ is the combined stiffness of all other structures, e.g., the polishing pad and polishing compound fluid, and $P_{\text {nominal }}$ is the nominal polishing pressure under the tool.

Here, we assume that $E^{\prime}$ is equivalent to the Young's modulus, because $E^{\prime}$ represents the elastic property of the Silly-Putty. The elastic stiffness $\kappa_{\text {elastic }}$ is proportional to $E^{\prime}$. The entire structure of the RC lap remains unchanged, except for variation of the elastic stiffness; therefore, the combined stiffness of all other components is a constant. Further, $\kappa_{\text {total }}$ can be expressed as a function of $V$ with

$$
k=\frac{\kappa_{\text {total }}(V)}{P_{\text {nominal }}} .
$$

Inspired by Burge, Kim, and Martin [17], the following differential equation can be derived directly from (1):

$$
\frac{d \varepsilon(Z)}{d Z}=\frac{d\left[\varepsilon(Z)-\varepsilon_{0}\right]}{d Z}=-k \cdot\left[\varepsilon(Z)-\varepsilon_{0}\right]
$$

having the solution

$$
\varepsilon(Z)=\left(\varepsilon_{\text {ini }}-\varepsilon_{0}\right) \cdot e^{-k \cdot Z}+\varepsilon_{0}
$$

where $\varepsilon$ is the $\mathrm{PV}$ magnitude of a particular spatial frequency error as a continuous function of the nominal removal depth $Z$. In order to transform $\varepsilon(Z)$ to a function of the polishing time $t$, we combine (8) and the well-known Preston equation

$$
Z=K_{p} \cdot P \cdot V \cdot t .
$$

Hence, we obtain

$$
\varepsilon(t)=\left(\varepsilon_{\text {ini }}-\varepsilon_{0}\right) \cdot e^{-k \cdot K_{p} \cdot P_{\text {nominal }} \cdot V \cdot t}+\varepsilon_{0}
$$

where $K_{p}$ is the coefficient of the Preston equation, $P$ is the polishing pressure, and $t$ is the polishing time. Using (6) and (10), the final mathematical model is expressed as

$$
\varepsilon(t)=\left(\varepsilon_{\text {ini }}-\varepsilon_{0}\right) \cdot e^{-\kappa_{\text {total }}(V) \cdot K_{p} \cdot V \cdot t}+\varepsilon_{0} .
$$

Equation (11) clearly indicates that the smoothing rate is related to $\kappa_{\text {total }}, K_{p}$, and $V$. We can divide the contribution of $V$ to the smoothing rate into two terms: $\kappa_{\text {total }}(V)$ and the removal term $K_{p} \cdot V$. The former is a non-analytic function of $V$, and the contribution of $V$ decreases if $V$ increases, based on the $E^{\prime}$ trend shown in Fig. 3. For the latter, it is apparent that $K_{p} \cdot V$ is related to $V$ directly. In other words, we can assume that an infinite smoothing rate will be obtained using an infinite $V$. Thus, $K_{p} \cdot V$ dominates $k$, and determining the limitation $V$ is the first task of our strategy. Our second task is to apply this limitation $V$ to actual smoothing experiments, so as to calculate $\kappa_{\text {total }}$ by fitting the data to the $S F$ function. Finally, we evaluate the combined influence on $k$ of $\kappa_{\text {total }}, K_{p}$, and $V$, based on our smoothing model. In addition, (11) is also very valuable for quantitative prediction of the smoothing effect for a real fabrication process. For a CCOS process, the dwell map is based on the de-convolution of the target removal map using a TIF. If we obtain this dwell map, we can estimate the MSF errors before polishing and the resultant change.

(2) Tool optimization

The smoothing is not an independent process, as it must be accompanied by the figuring. In other words, no technique to correct MSF errors without removing any large-scale errors has been developed in the history of CCOS. The use of a low-quality TIF to figure the surface is one of the MSF-error sources. Thus, the TIF is one of the key factors influencing the smoothing process, which further explains why we employ the RC lap as the polishing tool in this study.

The use of a lowered drive pin hole allows the original RC lap to overcome the gradient pressure effect, which is induced by the moment from the shear force on the workpiece surface [4]. When 
polishing hard ceramic materials such as $\mathrm{RB}-\mathrm{SiC}$, the polishing pressure is higher ( $>1 \mathrm{psi}$ ) than the conventional range (0.2 psi-0.6 psi). An unstable TIF occurs when higher polishing pressure is applied to the RC lap, which is caused by the local high pressure at the tool edge. A new RC-lap structure is designed herein, in order to mitigate the high nominal polishing pressure at the edge using a lowered back plate. Schematic 3-dimentional (3D) models of the old and new RC-lap designs are shown in Fig. 4.

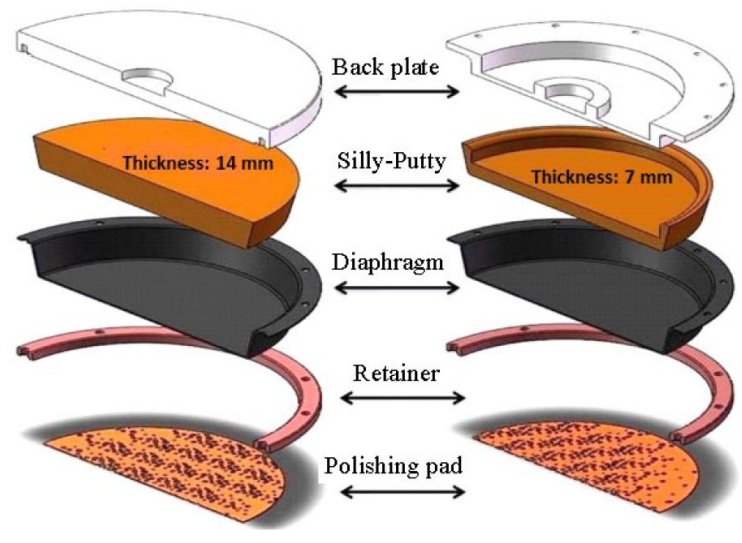

Fig. 4 3D schematic structures of old (left) and new (right) RC-lap structures (exploded and halved).

In order to understand the source of the high edge pressure, we consider an example using the static FEA to analyze the nominal contact pressure distribution between the tool and workpiece. The static elastic modulus of Silly-Putty is assumed to be $2 \mathrm{MPa}$ at $7 \mathrm{~Hz}$, based on the dynamic mechanical properties of this material [15]. For a $68-\mathrm{N}$ vertical force applied to the drive pin hole, symmetrical FEA models are established for the old and new RC laps $(100 \mathrm{~mm})$, using the ABAQUS software. The old (new) assembly model consists of 41592 (57797) nodes and 36292 (51874) elements, 35472 (50874) and 820 (1000) of which are linear hexahedral elements of type C3D8I and linear wedge elements of type C3D6, respectively. The FEA models and boundary conditions are shown in detail in Fig. 5. The RC-lap assembly-component material properties are listed in Table 1.

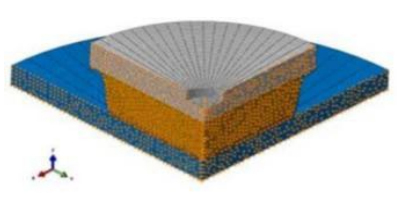

(a)

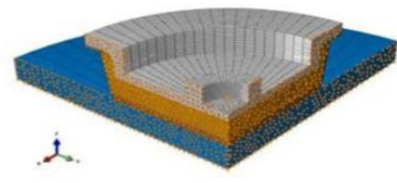

(b)
Fig. 5 Symmetrical FEA models for: (a) old and (b) new RC laps.

Table 1 Assembly-component material properties.

\begin{tabular}{cccc}
\hline Component & Material name & $\begin{array}{c}\text { Elasticmodulus } \\
\text { (MPa) }\end{array}$ & Poisson's ratio \\
\hline Back plate & Aluminum & $7.0 \mathrm{e} 4$ & 0.38 \\
$\begin{array}{c}\text { Non-Newton } \\
\text { fluid }\end{array}$ & Silly-Putty & 2 at 7 Hz & 0.45 \\
$\begin{array}{c}\text { Diaphragm } \\
\text { Pad }\end{array}$ & $\begin{array}{c}\text { Woven fabric reinforced } \\
\text { elastomer }\end{array}$ & 10 & 0.46 \\
Mirror & RBiversal LP-66 & 48 & 0.40 \\
\hline
\end{tabular}

The nominal contact pressure distribution between the RC lap and the workpiece is clearly shown in Fig. 6. An abrupt pressure change occurs at the edge in both cases. Because the RC lap consists of flexible materials, such as Silly-Putty, elastomer, and polyurethane, the high pressure at the edge is caused by the deformation of these materials when the vertical force to the drive pin hole becomes excessively high. Our new model minimizes this effect using a lowered back plate. The high edge pressure generates an undesirable TIF shape. Figure 7 depicts the orbital motion; the lap orbits the TIF center with a certain orbit radius. Ideally, the TIF peak region lies in the center, because the lap constantly covers the peak region in the figure. However, the line 1 in Fig. 7 indicates high pressure at the tool edge, which never affects the TIF peak region. Thus, the TIF peak region no longer corresponds to the TIF peak, and the line 2 indicates the new peak with a sharp cliff. Hence, a volcano-like TIF is obtained. Again, this effect is reduced by our proposed model, as shown below. Note that the real measured TIF may differ from the static FEA result, because the real motion is a dynamic problem. In addition, we have ignored the polishing compound fluid. 


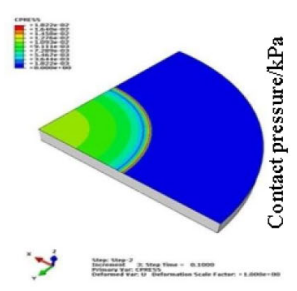

(a)

(a)

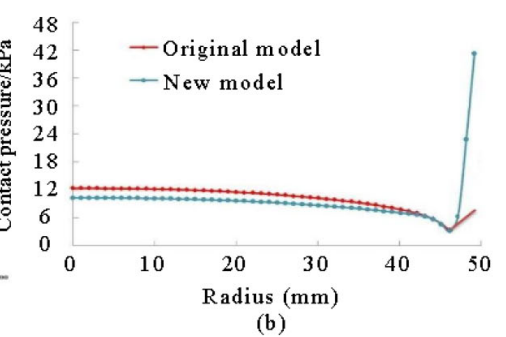

(b)
Fig. 6 Nominal contact pressure $P$ distribution: (a) 3D $P$ contour for new model and (b) $P$ distribution along workpieceradius for both old and new models.

In this study, we conducted two experiments using a $100-\mathrm{mm}$ RC lap at 300-RPM orbital speed, with a $15 \mathrm{~mm}$-orbital radius and under 1.47-psi polishing pressure; the results are shown in Fig. 8. The shape of the real measured TIF of our new structure was beyond our expectation, and a high, theory-like TIF was obtained.

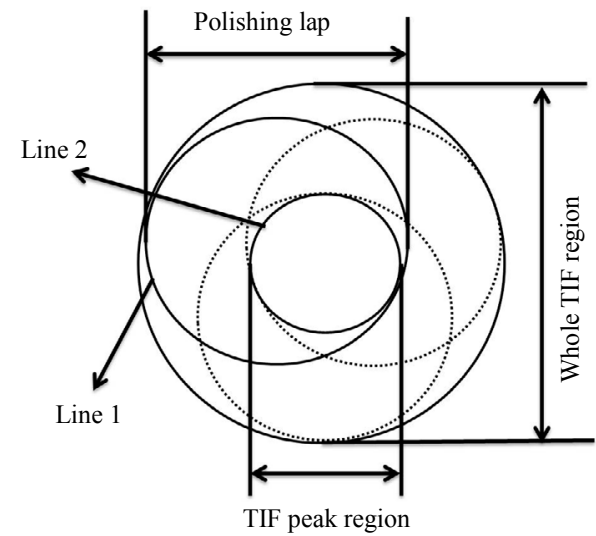

Fig. 7 Schematic picture of orbital motion (note: the line 1 and line 2 represent the high pressure at the lap edge and the edge of the TIF peak region, respectively; the dashed line indicates the lap position distribution region).
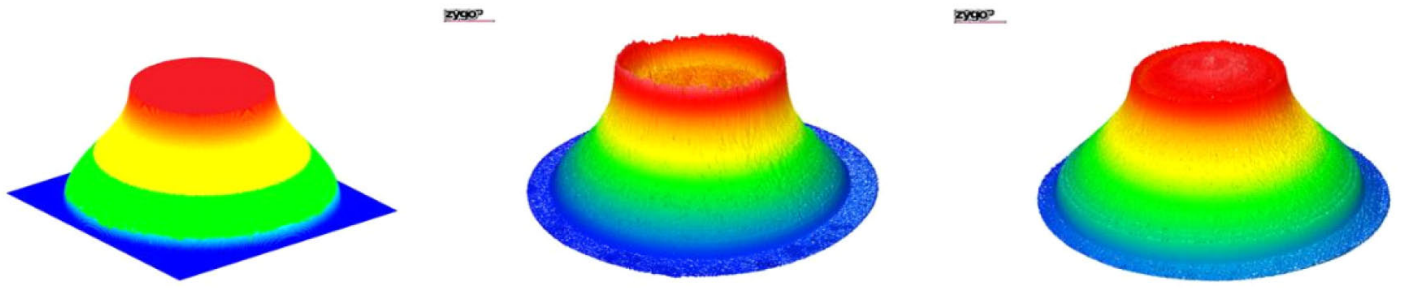

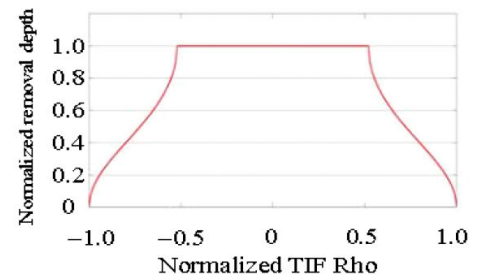

(a)

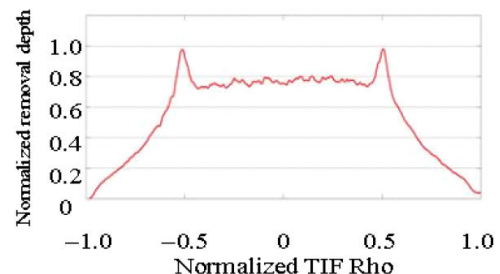

(b)

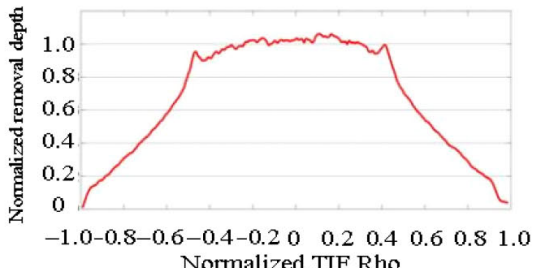

(c)

(a)

(b)

(c)

Fig. 8 3D measured TIFs (top) and their normalized radial profiles (bottom) for (a) theoretical, (b) old, and (c) new models.

\section{(3) Preston coefficient vs. tool speed}

As discussed in Section 3(1), the influence of $K_{p} \cdot V$ on the smoothing rate is greater than that of $\kappa_{\text {total }}(V)$. In this section, we discuss the $K_{p} \cdot V$ limitation using actual removal experiments.

For many CCOS processes, the material removal amount is calculated based on the well-known Preston equation given in (9). In order to determine the $K_{p} \cdot V$ limitation, the $K_{p}$ values for a wide range of $V$ (50 RPM-400 RPM) were measured based on the Preston equation. Forty experiments were performed to measure the TIFs, with the $K_{p}$ then being calculated from the TIFs. The detailed experimental conditions are listed in Table 2. The actual $100-\mathrm{mm}$ $\mathrm{RC}$ lap and a computer numerically controlled (CNC) polishing machine are shown in Figs. 9 and 10, respectively. 
Table 2 Overall experimental conditions.

\begin{tabular}{cc}
\hline Polishing tool & $100-\mathrm{mm} \mathrm{RC}$ lap \\
\hline Workpiece & 150 -mm RB-SiC \\
Polishing compound & Diamond slurry poly (2- $\mu \mathrm{m}$ particle \\
& size) \\
Tool motion & Orbital tool motion (15-mm orbital \\
& radius) \\
Tool motion speed range & 50 RPM-400 RPM (50-RPM interval) \\
Polishing pressure & $1.47 \mathrm{psi}$ \\
Repetitions & 5 \\
\hline
\end{tabular}

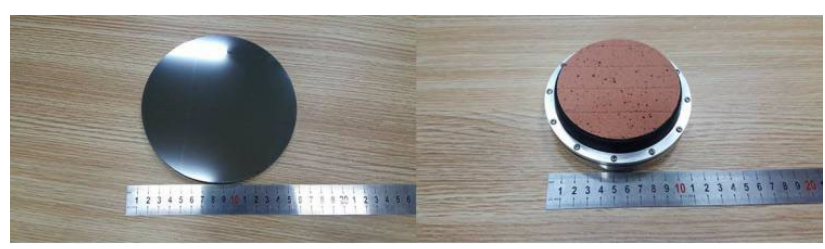

(a)

(b)

Fig. 9 Workpiece and polishing tool for experiments: (a) 150-mm RB-SiC and (b) 100-mm RC lap.

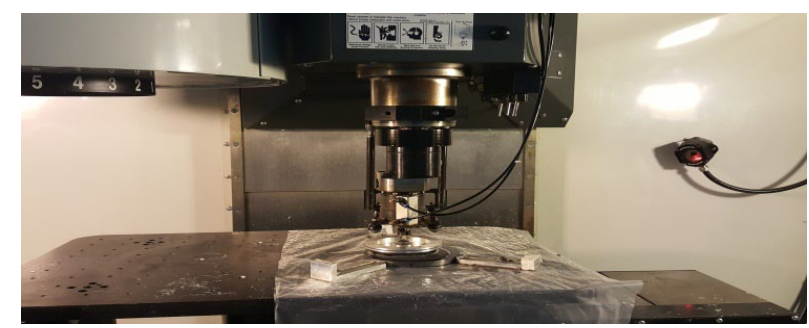

Fig. 10 CNC polishing machine used for experiments.

To ensure that there is no adverse impact on the surface roughness $R a$ from the high-speed orbital motion, it is necessary to measure the $R a$ values for all experiments. The average $R a$ values are plotted in Fig. 11, and the typical macro roughness of RB-SiC is shown in Fig. 12. The results indicate that $R a$ is insensitive to the tool speeds for all cases.

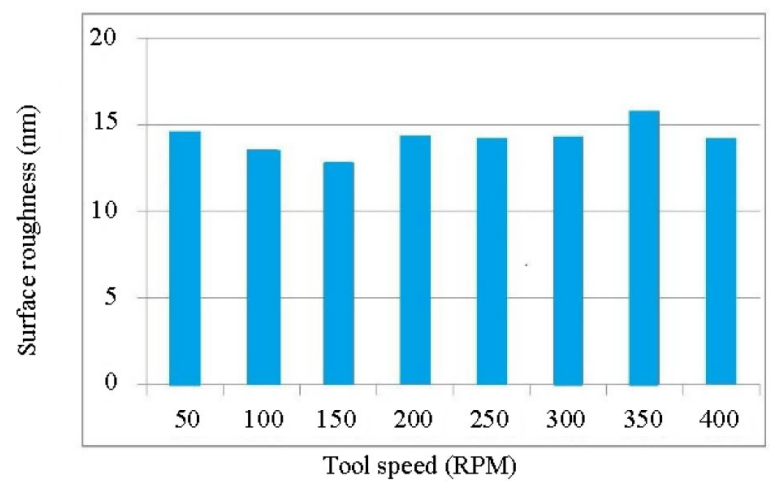

Fig. 11 Average surface roughness $R a$ values for all experiments.

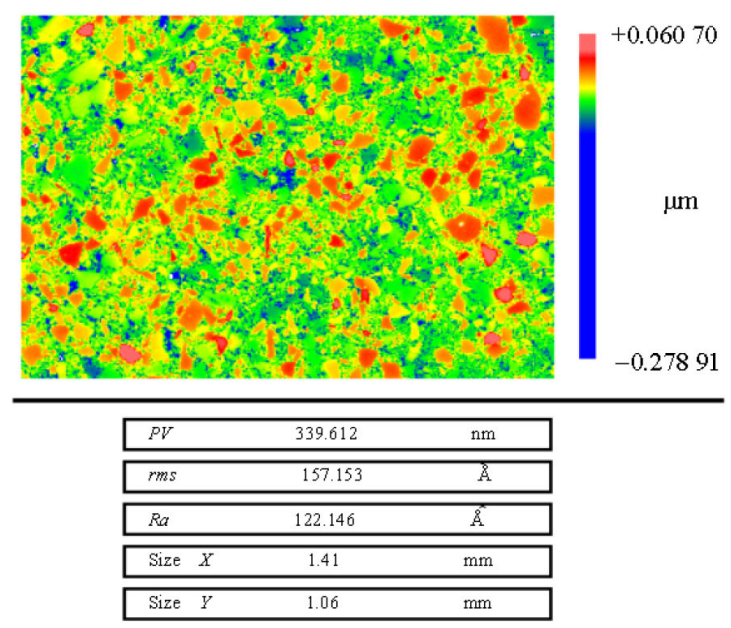

Fig. 12 RB-SiC roughness for 150-RPM orbital motion under 1.47-psi polishing pressure $P$.

The measured $K_{p}$ is plotted in Fig. 13. Each marker is the average value, and the standard deviation is indicated by the error bar. For a $V$ of 50-RPM, the $K_{p}$ is comparatively low. As $V$ increases in the 100-RPM - 300-RPM range, $K_{p}$ increases exponentially. However, for the $V$ range of $300 \mathrm{RPM}-400 \mathrm{RPM}, K_{p}$ decreases linearly. In order to clearly illustrate the relationship between the material removal and $V$, the $\Delta Z$ per hour is calculated (solid line, Fig. 13) using the averaged $K_{p}$ values. Hence, it is apparent that the material $Z$ increases only slightly as $V$ increases from $300 \mathrm{RPM}$ to $400 \mathrm{RPM}$, indicating that the smoothing rate $K_{p} \cdot V$ has reached a limitation. As stated above, our second task is to apply the limiting $V$ to actual smoothing experiments, so as to calculate $\kappa_{\text {total }}$ by fitting the data to the $S F$ function. As mentioned in Section $3(1), \kappa_{\text {total }}(V)$ is a non-analytic function.

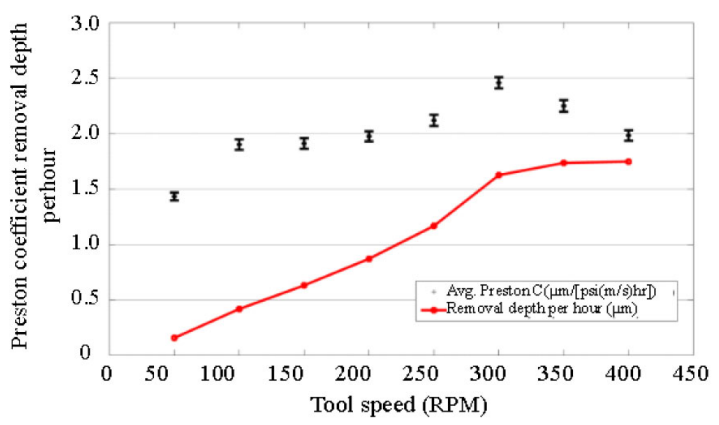

Fig. 13 Non-linearity of Preston coefficient $K_{p}$ over tool-speed $V$ range. 
Therefore, its contribution must be verified via smoothing experiments; these experiments are discussed in Section 4.

\section{Smoothing experiments and results}

(1) Experimental setup

In order to quantitatively calculate the compressive $\kappa_{\text {total }}(V)$, the parametric smoothing model in (1) was used. The linear trend was fitted with the experimental data $\left(\varepsilon_{0}, \Delta \varepsilon\right.$, and $\left.\Delta Z\right)$ to obtain the slope of the $S F$ function, i.e., $k$. Hence, $\kappa_{\text {total }}$ could be calculated from (6).

A sinusoidal ripple with $\xi=0.2 \mathrm{~mm}^{-1}$ and $P V=\sim 0.1 \mu \mathrm{m}$ was generated on the central areas of 150-mm diameter RB-SiC workpieces using magneto rheological finishing (MRF), as shown in Fig. 14. The reference area at the edge was intentionally left for the $\Delta Z$ measurement. Smoothing experiments were then conducted on these ripples.

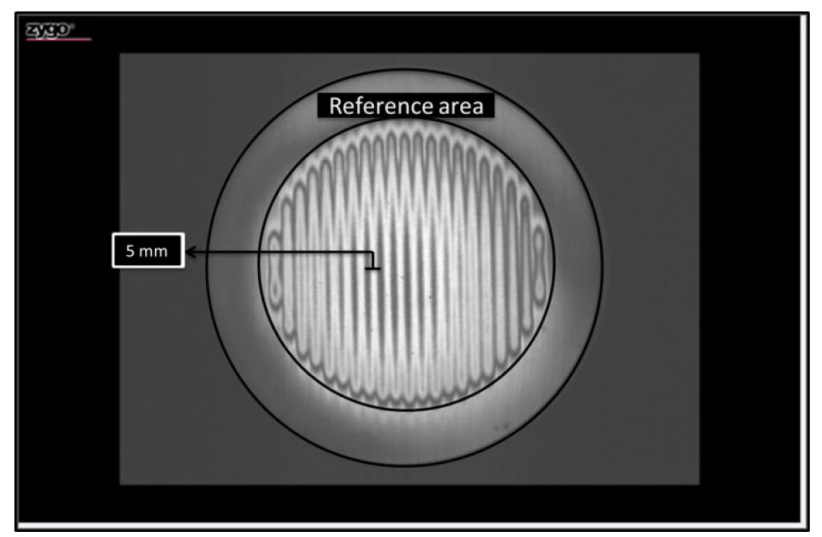

Fig. 14 Intensity map of MRF-generated sinusoidal ripples and reference area to measure nominal removal depth $\Delta Z$.

To facilitate a more informative comparison, two sets of experiments comparing the $\kappa_{\text {total }}$ obtained using 300-RPM and 400-RPM RC-lap orbital tool motions were performed. A Zygo Verifire ${ }^{\mathrm{TM}} \mathrm{QPZ}$ interferometer was used to monitor $\Delta \varepsilon$ and $\Delta Z$. The smoothing process was repeated until there was no obvious surface error reduction $\left(\varepsilon_{0}\right)$. Details of the experimental setup are provided in Table 3 .
Table 3 Experimental conditions for smoothing.

\begin{tabular}{ccc}
\hline & 300-RPM tool speed & 400-RPM tool speed \\
\hline Polishing tool & 100 -mm RC lap & 100-mm RC lap \\
Workpiece & 150-mm RB-SiC & 150-mm RB-SiC \\
Tool motion & $\begin{array}{c}\text { Orbital tool motion } \\
(15-\mathrm{mm} \text { orbital radius })\end{array}$ & $\begin{array}{c}\text { Orbital tool motion } \\
(15-\mathrm{mm} \text { orbital radius })\end{array}$ \\
Polishing pressure & 1.47 psi & 1.47 psi \\
Polishing compound & $\begin{array}{c}\text { Diamond slurry poly } \\
(2-\mu \mathrm{m} \text { particle size })\end{array}$ & $\begin{array}{c}\text { Diamond slurry poly } \\
(2-\mu \mathrm{m} \text { particle size })\end{array}$ \\
\hline
\end{tabular}

(2) Experimental results

Because some errors were induced during the smoothing processes, a band-pass (wavelength: $2 \mathrm{~mm}-6.5 \mathrm{~mm}$ ) fast Fourier transform (FFT) filter was applied to separate the MSF error information from the measured map. Some measured surface errors and the filtered data are presented as examples in Fig. 15. The averaged $P V$ value was determined from the filtered data to obtain the $\varepsilon_{0}, \Delta \varepsilon$, and $\Delta Z$ values, and then to fit the $S F$ function.

The $S F$ function was successfully fit using the experimental data, as shown in Fig. 16. From $k$, i.e., the slope of the $S F$ function, and (6), the RC-lap compressive $\kappa_{\text {total }}$ was calculated as listed in Table 4.

It is clearly apparent that the 400-RPM $V$ yields higher compressive $\kappa_{\text {total }}$, which is caused by the non-linear visco-elastic behavior of the Silly-Putty. In Section 3(1), it was explained that the final $k$ is related to $\kappa_{\text {total }}, V$, and $K_{p}$. Applying (11) to the $K_{p}$ measured in Section 3(3) and the $\kappa_{\text {total }}$ calculated from the $S F$ function, we can express the surface error as a function of the smoothing time $t$ directly. An example having a $0.3-\mu \mathrm{m} \varepsilon_{\mathrm{ini}}$ is shown in Fig. 17 to facilitate a comparison of the $k$ obtained for 300-RPM and 400-RPM $V$ values. 


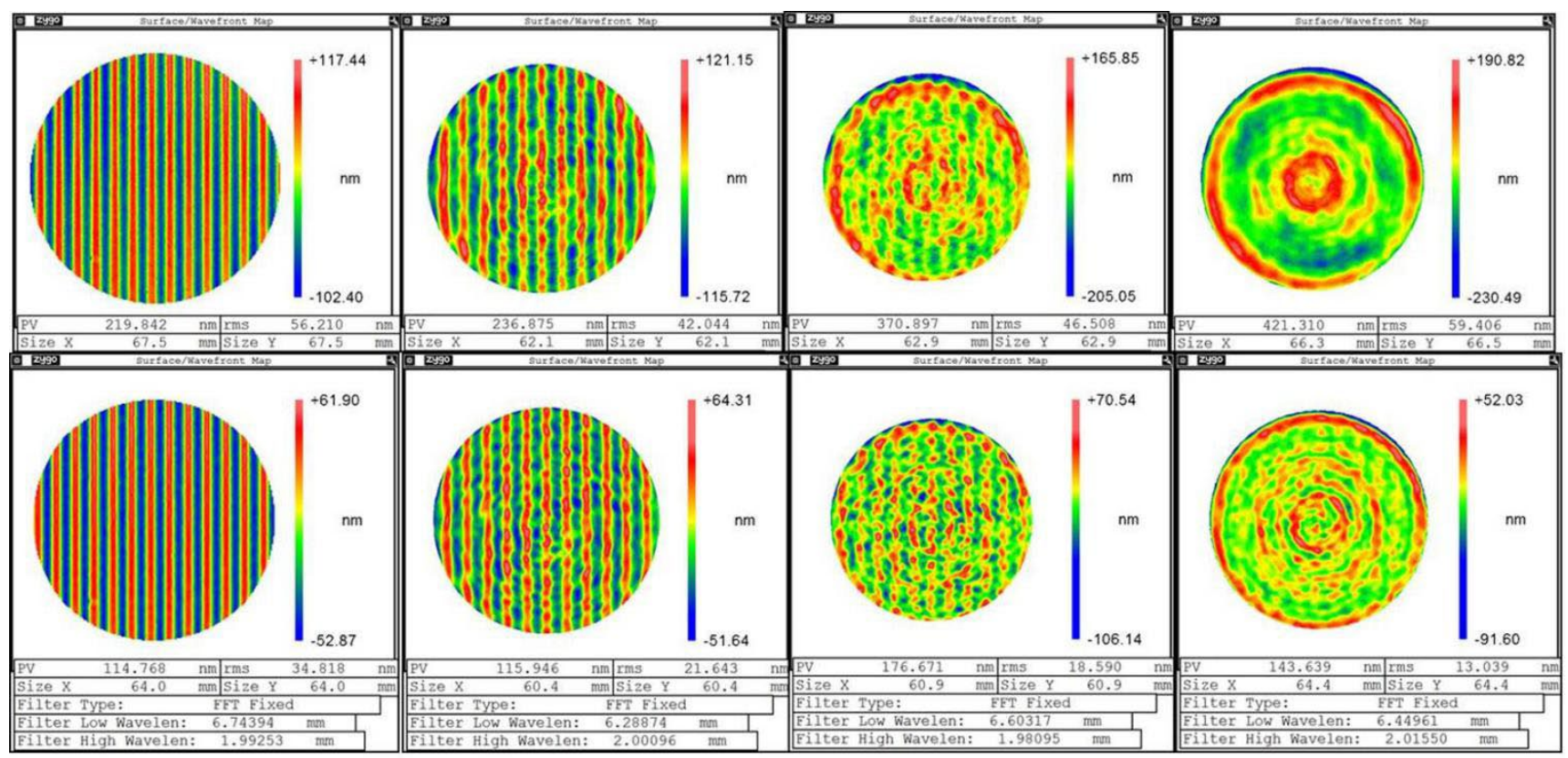

(a)

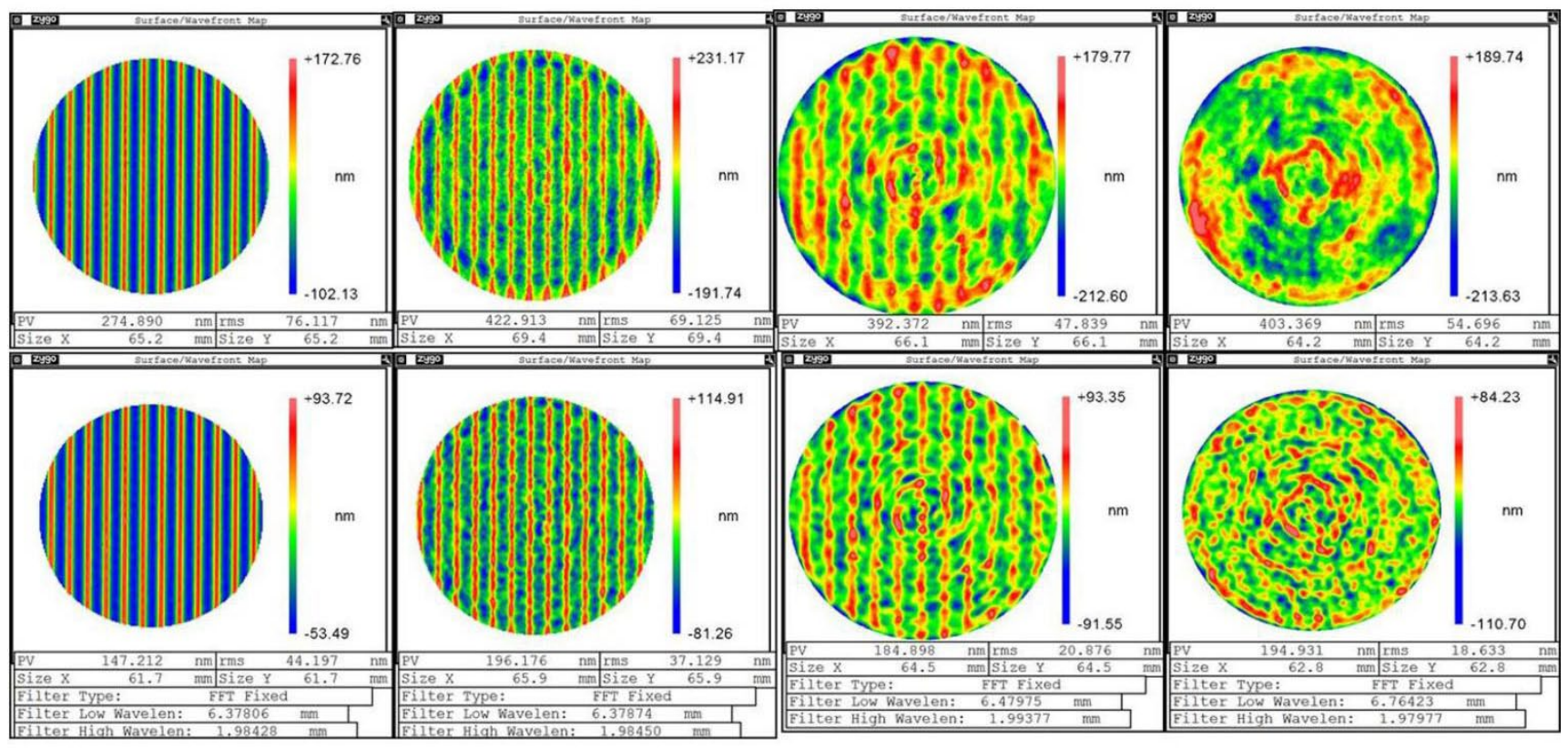

(b)

Fig. 15 Selected measured surface errors (top) and filtered data (bottom) in timed sequence: (a) 300-RPM and (b) 400-RPM tool-speed $V$ results. 


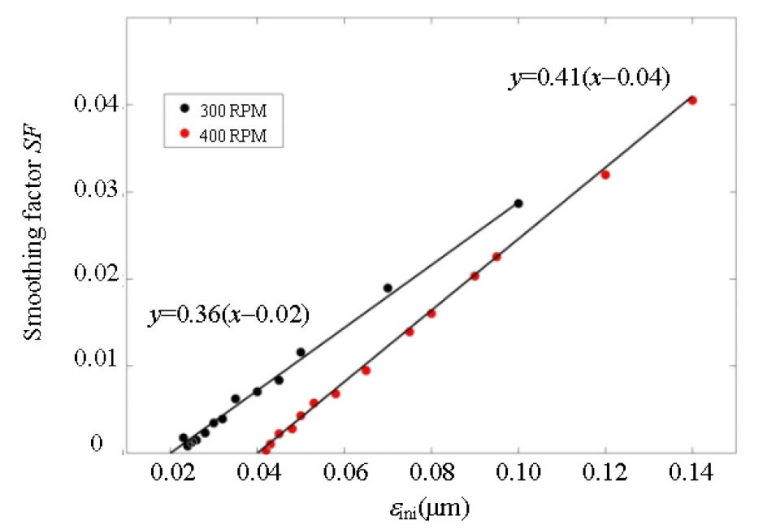

Fig. 16 Measured smoothing factor $S F v s$. initial magnitude $\varepsilon_{\text {ini }}$ and linear fitting results for 300-RPM and 400-RPM tool speed $V$.

Table 4 Slope of $S F$ function $k$ and compressive tool stiffness $\kappa_{\text {total }}$.

\begin{tabular}{ccc}
\hline $\mathrm{RPM}$ & 300 & 400 \\
\hline$k$ & 0.36 & 0.41 \\
$\kappa_{\text {total }}(\mathrm{Pa} / \mu \mathrm{m})$ & 3649 & 4054 \\
\hline
\end{tabular}

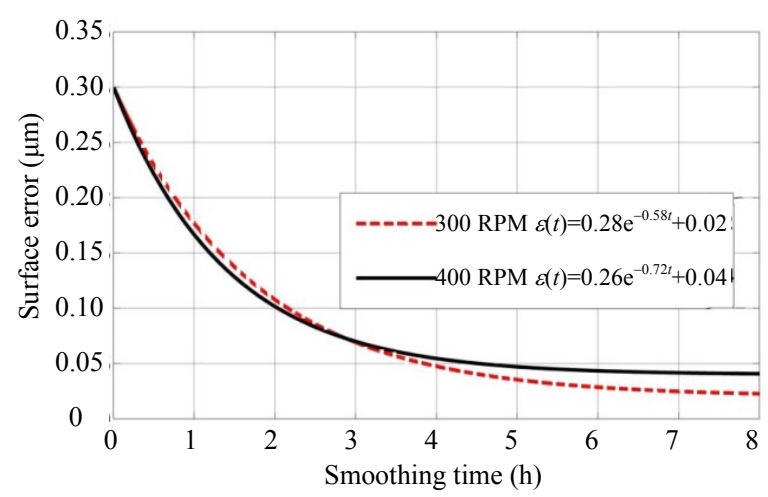

Fig. 17 Surface error $\varepsilon_{\text {ini }}$ vs. smoothing time $t$ for 300-RPM and 400-RPM tool speed $V$.

The result is straightforward. The ripples are almost fully smoothed out for both cases. The convergence rate is higher for the 400-RPM tool speed, but $\varepsilon_{0}$ for the 300 -RPM case is lower. Our actual data in Fig. 15 also explain this result. The error map for the 300-RPM case is smoother, having a 13.039-nm root-mean-squared (RMS) value in the final filtered data. In contrast, the RMS value of the 400-RPM case is $18.633 \mathrm{~nm}$. The smoothing rates for the 300-RPM and 400-RPM cases are almost identical, which verifies our assumption: the $K_{p} \cdot V$ term is more important than the $\kappa_{\text {total }}$ term for the RC laps. In addition, the induced errors of the 400-RPM case, which may be caused by the higher $\kappa_{\text {total }}$ or the higher $V$, yield a higher (i.e., less favorable) $\varepsilon_{0}$. Thus, the optimal smoothing speed $V$ considering $t$ is $300 \mathrm{RPM}$.

Note that the errors induced by the polishing parameters will be studied in a separate investigation in the future. Further, as shown in Fig. 18, the 300-mm RC lap was successfully employed in the Key Laboratory of Optical System Advanced Manufacturing Technology at the Changchun Institute of Optics, Fine Mechanics, and Physics (CIOMP).

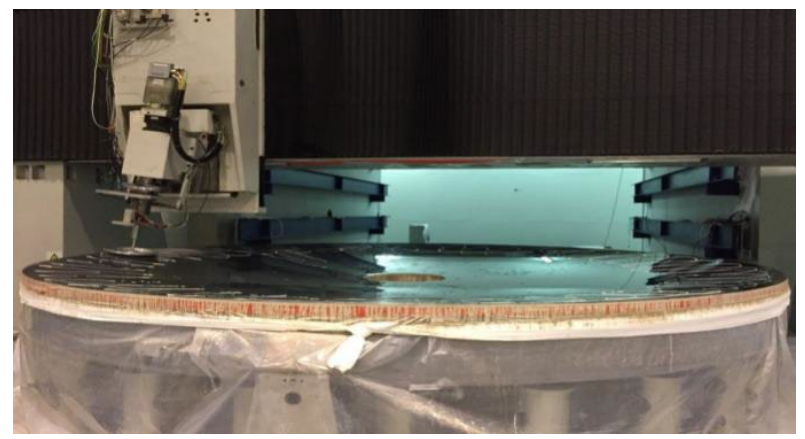

Fig. 18 300-mm diameter RC lap on 4-m-diameter RB-SiC mirror at CIOMP.

\section{Conclusions}

A time-dependent smoothing evacuation model that contains specific smoothing parameters was successfully derived from the parametric smoothing model and the Preston equation. Based on the time-dependent model, we proposed a strategy to improve the smoothing rate. A new RC-lap structure was designed to overcome the extreme polishing pressure, while providing a highly stable, theory-like TIF. Using our new structure, the limiting speed of 300-RPM was determined via a series of experiments. The surface roughness values were also measured for all experiments, being stable at $12 \mathrm{~nm}-15 \mathrm{~nm}$ for all cases. Two sets of smoothing experiments were performed using 300-RPM and 400-RPM tool speeds. The $S F$ function was successfully fit against the experimental data and the tool stiffness was calculated. Based on the 
time-dependent model, the smoothing rates for various tool speeds were compared. Hence, the optimal smoothing tool speed was defined as being 300-RPM

\section{Acknowledgment}

This research is financially supported by the National Natural Science of China (NSFC) (61210015) and Youth Foundation of National Natural Science Foundation (61605202).

Open Access This article is distributed under the terms of the Creative Commons Attribution 4.0 International License (http://creativecommons.org/ licenses/by/4.0/), which permits unrestricted use, distribution, and reproduction in any medium, provided you give appropriate credit to the original author(s) and the source, provide a link to the Creative Commons license, and indicate if changes were made.

\section{References}

[1] J. Nelson and G. H. Sanders, "The status of the thirty meter telescope project," SPIE, 2008, 7012: 70121A-1-70121A-18.

[2] M. Johns, P. Mccarthy, K. Raybould, A. Bouchez, A. Farahani, J. Filgueira, et al., "Giant magellan telescope: overview," SPIE, 2012, 8444: 84441H-184441H-16.

[3] T. Hull, M. J. Riso, J. M. Barentine, and A. Magruder, "Mid-spatial frequency matters: examples of the control of the power spectral density and what that means to the performance of imaging systems," SPIE, 2012, 8353: 835329-1-835329-17.

[4] D. W. Kim and J. H. Burge, "Rigid conformal polishing tool using non-linear visco-elastic effect," Optics Express, 2010, 18(3): 2242-2257.

[5] H. M. Martin, D. S. Anderson, J. R. P. Angel, R. H. Nagel, S. C. West, and R. S. Young, "Progress in the stressed-lap polishing of a 1.8-mf/1 mirror," SPIE, 1990, 1236: 682-690.

[6] J. H. Burge, B. Anderson, S. Benjamin, M. K. Cho,
K. Z. Smith, and M. J. Valente, "Development of optimal grinding and polishing tools for aspheric surfaces," SPIE, 1990, 4451: 153-164.

[7] Y. Shu, X. Nie, F. Shi, and S. Li, "Compare study between smoothing efficiencies of epicyclic motion and orbital motion," Optik - International Journal for Light and Electron Optics, 2014, 125(16): 4441-4445.

[8] N. J. Brown, P. C. Baker, and R. E. Parks, "Polishing-to-figuring transition in turned optics," Contemporary Methods of Optical Fabrication, 1981, 306(6): 58-65.

[9] R. A. Jones, "Computer simulation of smoothing during computer-controlled optical polishing," Applied Optics, 1995, 34(7): 1162-1169.

[10] P. K. Mehta and P. B. Reid, "Mathematical model for optical smoothing prediction of high-spatialfrequency surface errors," SPIE, 1999, 3786: 447-459.

[11] M. T. Tuell, J. H. Burge, and B. Anderson. "Aspheric optics: Smoothing the ripples with semi-flexible tools," Optical Engineering, 2002, 15(2): 1473-1474.

[12] D. W. Kim, W. H. Park, H. K. An, and J. H. Burge, "Parametric smoothing model for visco-elastic polishing tools," Optics Express, 2010, 18(21): 22515-22526.

[13] Y. Shu, D. W. Kim, H. M. Martin, and J. H. Burge, "Correlation-based smoothing model for optical polishing," Optics Express, 2013, 21(23): 28771-28782.

[14] Y. Shu, X. Nie, F. Shi, and S. Li, "Smoothing evolution model for computer controlled optical surfacing," Journal of Optical Technology, 2014, 81(3): 164-167.

[15] X. Nie, S. Li, F. Shi, and H. Hu, "Generalized numerical pressure distribution model for smoothing polishing of irregular midspatial frequency errors," Applied Optics, 2014, 53(6): 1020-1027.

[16] A. C. Fischer-Cripps, "Multiple-frequency dynamic nanoindentation testing," Journal of Materials Research, 2004, 19(19): 2981-2988.

[17] J. H. Burge, D. W. Kim, and H. M. Martin, "Process optimization for polishing large aspheric mirrors,” SPIE, 2014, 9151: 91512R-191512R-13. 